\title{
Contractive Homomorphisms and Tensor Product Norms
}

\author{
Bhaskar Bagchi and Gadadhar Misra
}

For any complex domain $\Omega$, one can ask if all contractive algebra homomorphisms of $\mathcal{A}(\Omega)$ (into the algebra of Hilbert space operators) are completely contractive or not. By Ando's Theorem, this has an affirmative answer for $\Omega=\mathbb{D}^{2}$, the bi-disc - while the answer is unknown for $\Omega=\left(\ell^{1}(2)\right)_{1}$, the unit ball of $\mathbb{C}^{2}$ with $\ell^{1}$ - norm. In this paper, we consider a special class of homomorphisms associated with any bounded complex domain; this well known construct generalizes Parrott's example. Our question has an affirmative answer for homomorphisms in this class with $\Omega=\left(\ell^{1}(2)\right)_{1}$. We show that there are many domains in $\mathbb{C}^{2}$ for which the question can be answered in the affirmative by reducing it to that of $\Omega=\mathbb{D}^{2}$ or $\left(\ell^{1}(2)\right)_{1}$. More generally, the question for an arbitrary $\Omega$ can often be reduced to the case of the unit ball of an associated finite dimensional Banach space. If we restrict attention to a smaller subclass of homomorphisms the question for a Banach ball becomes equivalent to asking whether in the analogue of Grothendieck's inequality, in this Banach space, restricted to positive operators, the best constant is $=1$ or not. We show that this is indeed the case for $\Omega=\mathbb{D}^{2}, \mathbb{D}^{3}$ or the dual balls, but not for $\mathbb{D}^{n}$ or its dual for $n \geq 4$. Thus we isolate a large class of homomorphisms of $\mathcal{A}\left(\mathbb{D}^{3}\right)$ for which contractive implies completely contractive. This has many amusing relations with injective and projective tensor product norms and with Parrott's example.

\section{Contractive Homomorphisms}

Let $\Omega \subseteq \mathbb{C}^{m}$ be a bounded domain, and $\mathbb{C}^{n \times n}$ be the $n \times n$ matrices over the complex field. For $\omega$ in $\Omega$ and $A_{1}, \ldots, A_{m}$ in $\mathbb{C}^{n \times n}$, let

$$
\langle\nabla f(\omega), \boldsymbol{A}\rangle=A_{1} \frac{\partial f}{\partial z_{1}}(\omega)+\cdots+A_{m} \frac{\partial f}{\partial z_{m}}(\omega), \quad f \in H^{\infty}(\Omega),
$$

where $\boldsymbol{A}=\left(A_{1}, \ldots, A_{m}\right)$. The map $\varphi_{\omega}(\boldsymbol{A}, \cdot): H^{\infty}(\Omega) \rightarrow \mathbb{C}^{2 n \times 2 n}$, defined by

$$
\varphi_{\omega}(\boldsymbol{A}, f)=\left(\begin{array}{cc}
f(\omega) I_{n} & \langle\nabla f(\omega), \boldsymbol{A}\rangle \\
0 & f(\omega) I_{n}
\end{array}\right)
$$


is easily seen to be an unital algebra homomorphism, which is continuous from $H^{\infty}(\Omega)$ equipped with the topology of uniform convergence on compact sets into $\mathbb{C}^{2 n \times 2 n}$ with the usual operator norm topology. In this paper, we study a stronger notion of continuity. Indeed, we investigate the norm of each of the operators

$$
\varphi_{\omega}^{(k)}(\boldsymbol{A}, \cdot)=\varphi_{\omega}(\boldsymbol{A}, \cdot) \otimes I: H^{\infty}(\Omega) \otimes \mathbb{C}^{k \times k} \rightarrow\left(\mathbb{C}^{2 n \times 2 n} \otimes \mathbb{C}^{k \times k} \cong \mathbb{C}^{2 n k \times 2 n k}, \mathrm{op}\right),
$$

where $\|F\|=\sup \left\{\|F(z)\|_{\text {op }}: z \in \Omega\right\}$, for $F \in H^{\infty}(\Omega) \otimes \mathbb{C}^{k \times k}$. Let

$$
\left\|\varphi_{\omega}(\boldsymbol{A}, \cdot)\right\|_{\mathrm{cb}}=\lim _{k \rightarrow \infty}\left\|\varphi_{\omega}^{(k)}(\boldsymbol{A}, \cdot)\right\| .
$$

The map $\varphi_{\omega}(\boldsymbol{A}, \cdot)$ is said to be contractive if $\left\|\varphi_{\omega}(\boldsymbol{A}, \cdot)\right\| \leq 1$ and is completely contractive if $\left\|\varphi_{\omega}(\boldsymbol{A}, \cdot)\right\|_{\mathrm{cb}} \leq 1$.

It is an important open problem to determine domains $\Omega \subseteq \mathbb{C}^{m}$ for which every contractive homomorphism $\varphi_{\omega}(\boldsymbol{A}, \cdot)$ is completely contractive.

These homomorphisms are related to the familiar notion of a spectral set and complete spectral set for the commuting operator tuple

$$
\boldsymbol{N}(\omega, \boldsymbol{A})=\left(\left(\begin{array}{cc}
\omega_{1} I_{n} & A_{1} \\
0 & \omega_{1} I_{n}
\end{array}\right), \ldots,\left(\begin{array}{cc}
\omega_{m} I_{n} & A_{m} \\
0 & \omega_{m} I_{n}
\end{array}\right)\right)
$$

Thus, for any rational function $r$ in the algebra $\mathcal{R}(\bar{\Omega})$ of rational functions with poles off $\bar{\Omega}$, the evaluation map $r \rightarrow r(\boldsymbol{N}(\omega, \boldsymbol{A})), r \in \mathcal{R}(\bar{\Omega})$ is well defined and coincides with the homomorphism $\varphi_{\omega}(\boldsymbol{A}, \cdot)$ on $\mathcal{R}(\bar{\Omega})$.

Here are two competing definitions of spectral set (resp. complete spectral set). We would say that the operator tuple $\boldsymbol{N}(\omega, \boldsymbol{A})$ admits the compact set $\bar{\Omega}$ as a spectral set (resp. complete spectral) set if

1. the homomorphism $\varphi_{\omega}(\boldsymbol{A}, \cdot)$ is contractive ( resp. completely contractive ) on the algebra $\mathcal{A}(\bar{\Omega})$ of functions holomorphic in a neighbourhood of $\bar{\Omega}$, or if

2. the homomorphism $\varphi_{\omega}(\boldsymbol{A}, \cdot)$ is contractive ( resp. completely contractive ) on the algebra $\mathcal{R}(\bar{\Omega})$.

Agler [1] uses the first definition, while Paulsen [7] uses the second one.

These two notions of a spectral set need not coincide. We will be mainly concerned with the homomorphism $\varphi_{\omega}(\boldsymbol{A}, \cdot)$ as a map on $H^{\infty}(\Omega)$. Agler [1] points out that if $\varphi_{\omega}(\boldsymbol{A}, \cdot)$ is contractive over the algebra $H^{\infty}(U)$ for every open set $U$ containing $\bar{\Omega}$, then $\varphi_{\omega}(\boldsymbol{A}, \cdot)$ is 
contractive over $\mathcal{A}(\bar{\Omega})$. However, for certain domains, if the homomorphism $\varphi_{\omega}(\boldsymbol{A}, \cdot)$ or for that matter $\varphi_{\omega}^{(k)}(\boldsymbol{A}, \cdot)$ is contractive on any one of these algebras then it is contractive on all of them.

If $F=\left(f_{i j}\right) \in H^{\infty}(\Omega) \otimes \mathbb{C}^{k \times k}$, then the map $F: \Omega \rightarrow \mathbb{C}^{k \times k}, F(z)=\left(f_{i j}(z)\right)$ is holomorphic. Let

$$
F(z)=F(\omega)+\left(z_{1}-\omega_{1}\right) \frac{\partial F}{\partial z_{1}}(\omega)+\cdots+\left(z_{m}-\omega_{m}\right) \frac{\partial F}{\partial z_{m}}(\omega)+\cdots,
$$

be the power series expansion of $F$ around the point $\omega$ in $\Omega$ with matrix co-efficients. Thus

$$
\begin{aligned}
\varphi_{\omega}^{(k)}\left(\boldsymbol{A},\left(f_{i j}\right)\right) & =\left(\varphi_{\omega}\left(\boldsymbol{A}, f_{i j}\right)\right) \\
& =\left(\begin{array}{cc}
f_{i j}(\omega) I_{n} & \left\langle\nabla f_{i j}(\omega), \boldsymbol{A}\right\rangle \\
0 & f_{i j}(\omega) I_{n}
\end{array}\right) \\
& \sim\left(\begin{array}{cc}
F(\omega) \otimes I_{n} & \langle D F(\omega), \boldsymbol{A}\rangle \\
0 & F(\omega) \otimes I_{n}
\end{array}\right),
\end{aligned}
$$

where $\sim$ means that we have obtained the last matrix after elementary row and column operation from the preceeding one and

$$
\langle D F(\omega), \boldsymbol{A}\rangle=\frac{\partial F}{\partial z_{1}}(\omega) \otimes A_{1}+\cdots+\frac{\partial F}{\partial z_{m}}(\omega) \otimes A_{m} .
$$

There is a bi-holomorphic automorphism ( Möbius map ) of the matrix unit ball taking $F(\omega)$ to 0 , which is in fact a rational function. Using this map and the von Neumann inequality, it is not hard to prove the following theorem (cf. [6, 7]).

For a normed linear space $V$, let $(V)_{1}$ denote the open unit ball in $V$.

TheOREm $1.1\left\|\varphi_{\omega}^{(k)}(\boldsymbol{A}, \cdot)\right\| \leq 1$ if and only if $\|\langle D F(\omega), \boldsymbol{A}\rangle\| \leq 1$, for all holomorphic functions $F: \Omega \rightarrow\left(\mathbb{C}^{k \times k}\right)_{1}, F(\omega)=0$.

Thus, the homomorphism $\varphi_{\omega}(\boldsymbol{A}, \cdot)$ is contractive on $H^{\infty}(\Omega)$ if and only if

$$
\sup \left\{\|\langle\nabla f(\omega), \boldsymbol{A}\rangle\|_{\text {op }}: f \in \operatorname{Hol}_{\omega}(\Omega, \mathbb{D})\right\} \leq 1
$$

where $\mathbb{D}=\{z:|z| \leq 1\}$ and $\operatorname{Hol}_{\omega}(\Omega, \mathbb{D})=\{f: f: \Omega \rightarrow \mathbb{D}$ is holomorphic,$f(\omega)=0\}$. However the set

$$
\mathcal{D} \Omega(\omega)=\left\{\nabla f(\omega): f \in \operatorname{Hol}_{\omega}(\Omega, \mathbb{D})\right\}
$$

is a unit ball with respect to some norm in $\mathbb{C}^{m}$ ( see [7] ). The Carathéodory norm is the dual norm

$$
C_{\Omega, \omega}(\boldsymbol{v})=\sup \left\{|\langle\nabla f(\omega), \boldsymbol{v}\rangle|: f \in \operatorname{Hol}_{\omega}(\Omega, \mathbb{D})\right\}
$$


Proposition $1.2 \varphi_{\omega}(\boldsymbol{A}, \cdot)$ is contractive on $H^{\infty}(\Omega)$ if and only if $\varphi_{0}(\boldsymbol{A}, \cdot)$ is contracive on $H^{\infty}\left(\left(\mathbb{C}^{m}, C_{\Omega, \omega}\right)_{1}\right)$.

Proof: The condition in 1.1 for $k=1$ is equivalent to contractivity of the operator $L_{\boldsymbol{A}}$ : $\left(\mathbb{C}^{m}, C_{\Omega, \omega}^{*}\right) \rightarrow\left(\mathbb{C}^{n \times n}\right.$, op $)$, defined by

$$
L_{\boldsymbol{A}}(\lambda)=\lambda_{1} A_{1}+\cdots \lambda_{m} A_{m} \text { for } \lambda \in \mathbb{C}^{m} .
$$

Therefore, putting $\Sigma=\left(\mathbb{C}^{m}, C_{\Omega, \omega}\right)_{1}$, it suffices to verify the following claim: $\left(\mathbb{C}^{m}, C_{\Omega, \omega}\right)_{1}=$ $\left(\mathbb{C}^{m}, C_{\Sigma, 0}\right)_{1}$. Let $\mathcal{B}$ be a bounded domain in $\mathbb{C}^{m}$, which contains 0 and is balanced. If $f: \mathcal{B} \rightarrow \mathbb{D}$ is holomorphic, then $\nabla f(0): \mathcal{B} \rightarrow \mathbb{D}$ in accordance with the Schwarz lemma (see $[9$, page 161]). This implies that for any such domain $\mathcal{B}$, we have (in terms of notation in $(1.2))$

$$
\mathcal{D B}(0)=\{\ell: \mathcal{B} \rightarrow \mathbb{D}: \ell \text { is linear }\}
$$

Hence, if $\mathcal{B}$ is a ball with respect to some norm, then $\mathcal{D B}(0)$ is the dual ball. Applying this observation to the ball $\Sigma$ proves the claim.

Similarly, the homomorphism $\varphi_{\omega}^{(k)}(\boldsymbol{A}, \cdot)$ is contractive if and only if

$$
\sup \left\{\|\langle D F(\omega), \boldsymbol{A}\rangle\|_{\text {op }}: F \in \operatorname{Hol}_{\omega}\left(\Omega,\left(\mathbb{C}^{k \times k}\right)_{1}\right)\right\} \leq 1 .
$$

Again, the set

$$
\mathcal{D} \Omega^{(k)}(\omega)=\left\{D F(\omega): F \in \operatorname{Hol}_{\omega}\left(\Omega,\left(\mathbb{C}^{k \times k}\right)_{1}\right)\right\}
$$

is a unit ball with respect to some norm in $\mathbb{C}^{k \times m k}$ ( $\operatorname{see}[7]$ ). If we let $C_{\Omega, \omega}^{(k)}$ be the dual norm, then as before the condition in theorem 1.1 is equivalent to contractivity of the operator $L_{\boldsymbol{A}}^{(k)}:\left(\mathbb{C}^{k \times m k}, C_{\Omega, \omega}^{(k)^{*}}\right) \rightarrow\left(\mathbb{C}^{n k \times n k}\right.$, op $)$, defined by

$$
L_{\boldsymbol{A}}^{(k)}(\Lambda)=\Lambda_{1} \otimes A_{1}+\cdots+\Lambda_{m} \otimes A_{m}, \text { for } \Lambda \in \mathbb{C}^{m k \times m k} .
$$

An explicit description of the set $\mathcal{D} \Omega^{(k)}(\omega)$ will certainly help understand the operator norm of $L_{\boldsymbol{A}}^{(k)}$. In this note, we will describe the set $\mathcal{D} \Omega^{(k)}(\omega)$ for a product domain and a balanced domain.

Following Paulsen [7], we define for each domain $\Omega$, and a fixed point $\omega \in \Omega$, a numerical constant as follows

$$
\alpha(\Omega, \omega)=\sup \left\{\left\|\varphi_{\omega}(\boldsymbol{A}, \cdot)\right\|_{\mathrm{cb}}:\left\|\varphi_{\omega}(\boldsymbol{A}, \cdot)\right\| \leq 1, \varphi_{\omega}(\boldsymbol{A}, \cdot): H^{\infty}(\Omega) \rightarrow \mathbb{C}^{n \times n}\right\} .
$$

Note that $\alpha(\Omega, \omega)=\alpha(\hat{\Omega}, \omega)$, where $\hat{\Omega}$ is the holomorphic convex hull of $\Omega$. Therefore, $\hat{\Omega}$ is a domain of holomorphy, and we assume through out this paper that the domain $\Omega$ is a domain of holomorphy. 


\subsection{The CASE OF A BALANCED DOMAIN}

Let $\mathcal{B}$ be a bounded domain in $\mathbb{C}^{m}$, which contains zero and is balanced.

REMARK 1.3 We note that if we use rational functions or functions holomorphic in neighbourhood of $\bar{\Omega}$ in defining the set $\mathcal{D B}(0)$, we would still have

$$
\mathcal{D B}(0)=\{\ell: \mathcal{B} \rightarrow \mathbb{D}: \ell \text { is linear }\},
$$

and if $\mathcal{B}$ is a ball with respect to some norm, then

$$
\mathcal{D B}(0)=\left(\mathbb{C}^{m},\|\cdot\|_{\mathcal{B}}^{*}\right)_{1} .
$$

In particular, the operator norm on $\mathbb{C}^{k \times k}$ and the Carathéodory norm on $\left(\mathbb{C}^{k \times k}\right)_{1}$ at 0 coincide. Since holomorphic maps are norm decreasing with respect to the Carathéodory norm, it follows that for any holomorphic map $F \in \operatorname{Hol}_{0}\left(\mathrm{~B},\left(\mathbb{C}^{k \times k}, \text { op }\right)_{1}\right)$, the derivative

$$
D F(\omega)=\left(\frac{\partial F}{\partial z_{1}}(\omega), \ldots, \frac{\partial F}{\partial z_{m}}(\omega)\right):\left(\mathbb{C}^{m}, C_{\mathcal{B}, 0}\right) \rightarrow\left(\mathbb{C}^{k \times k}, \text { op }\right)
$$

is a contraction.

Lemma 1.4 If $\mathcal{B}$ is a bounded balanced domain in $\mathbb{C}^{m}$ then $\varphi_{0}(\boldsymbol{A}, \cdot)$ is completely contractive on $H^{\infty}(\mathcal{B})$ if and only if $\varphi_{0}(\boldsymbol{A}, \cdot)$ is completely contractive on $H^{\infty}\left(\left(\mathbb{C}^{m}, C_{\mathcal{B}, 0}\right)_{1}\right)$

Proof: By the preceeding remark,

$$
\mathcal{D B}^{(k)}(0) \subseteq\left(\left(\mathbb{C}^{m}, C_{\mathcal{B}, 0}^{*}\right) \check{\otimes}\left(\mathbb{C}^{k \times k}, \mathrm{op}\right)\right)_{1}
$$

Since the convex hull $c o \mathcal{B}$ of the balanced region $\mathcal{B}$ is a ball, it follows that

$$
\begin{aligned}
\mathcal{D B}(0)=\left(\mathbb{C}^{m}, C_{\mathcal{B}, 0}^{*}\right)_{1} & =\{\ell: \mathcal{B} \rightarrow \mathbb{D}: \ell \text { is linear }\} \\
& =\{\ell: \operatorname{co} \mathcal{B} \rightarrow \mathbb{D}: \ell \text { is linear }\}=\left(\mathbb{C}^{m}, C_{\text {co B }, 0}^{*}\right)_{1}
\end{aligned}
$$

(see 1.8). For $\Lambda_{1}, \ldots, \Lambda_{m} \in \mathbb{C}^{k \times k}$, define the linear map $\Lambda:\left(\mathbb{C}^{m}, C_{\mathcal{B}, 0}\right) \rightarrow\left(\mathbb{C}^{k \times k}\right.$, op $)$ by

$$
\Lambda(z)=z_{1} \Lambda_{1}+\cdots+z_{m} \Lambda_{m} .
$$

Any such linear contraction is holomorphic and maps $\mathcal{B}$ into $\left(\mathbb{C}^{k \times k}, \text { op }\right)_{1}$. Therefore, we have the opposite inequality in 1.10 . The contractivity of $\varphi_{\omega}^{(k)}(\boldsymbol{A}, \cdot)$ is thus equivlent to

$$
\sup \left\{\left\|\Lambda_{1} \otimes A_{1}+\cdots+\Lambda_{m} \otimes A_{m}\right\|_{\text {op }}: \Lambda \in\left(\mathbb{C}^{m}, C_{\mathcal{B}, 0}^{*}\right) \check{\otimes}\left(\mathbb{C}^{k \times k}, \text { op }\right)_{1}\right\} \leq 1 .
$$

The simple observation (see 1.9) that

$$
\mathcal{D}\left(\left(\mathbb{C}^{m}, C_{\mathcal{B}, 0}\right)_{1}\right)^{(k)}(0)=\left(\left(\mathbb{C}^{m}, C_{\mathcal{B}, 0}^{*}\right) \check{\otimes}\left(\mathbb{C}^{k \times k}, \text { op }\right)\right)_{1}=\mathcal{D} \mathcal{B}^{(k)}(0)
$$

completes the proof. 
EXAMPLE 1.5 Let $0<r \leq 1 / 4$, and

$$
\mathcal{B}=\left\{\boldsymbol{z}=\left(z_{1}, z_{2}\right) \in \mathbb{C}^{2}:\left|z_{1}\right|+\left|z_{2}\right|<1 \text { and }\left|z_{1}\right|\left|z_{2}\right|<r\right\}
$$

Since $\mathcal{B}$ is a Rienhardt domain, and is logarithmically convex, it follows that it is a domain of holomorphy. Remark 1.3 implies that $\mathcal{D} \mathcal{B}(0)$ is the unit bi-disk. Therefore, $\left(\mathbb{C}^{2}, C_{\mathcal{B}, 0}\right)$ is just the unit ball in $\mathbb{C}^{2}$ with respect to the $\ell^{1}$ norm. By lemma $1.4, \alpha(\mathcal{B}, 0)=\alpha\left(\left(\mathbb{C}^{m}, C_{\mathcal{B}, 0}\right)_{1}, 0\right)$. Ando's theorem (cf. [7]) and the duality result from [6] shows that $\alpha(\mathcal{B}, 0)=1$.

Note that this is a genuinely new example of a domain $\mathcal{B}$ with $\alpha(\mathcal{B}, 0)=1$ since the Remark [5, page 364] clearly implies that this domain is not bi-holomorphically equivalent to the unit ball of $\ell^{1}(2)$.

\subsection{The CASE OF AN ARBITRARY DOMAIN}

We now examine the relationship between the contractivity of

1. $\varphi_{\omega}^{(k)}(\boldsymbol{A}, \cdot)$ on the algebra $H^{\infty}(\Omega)$ and

2. $\varphi_{0}^{(k)}(\boldsymbol{A}, \cdot)$ on the algebra $H^{\infty}\left(\left(\mathbb{C}^{m}, C_{\Omega, \omega}\right)_{1}\right)$.

If $k=1$, then we have verified that these two questions are equivalent (Proposition 1.2) . The situation is more complicated for $k>1$.

THEOREM 1.6 If there is a holomorphic map $H: \Omega \rightarrow\left(\mathbb{C}^{m}, C_{\Omega, \omega}\right)_{1}$ such that

$$
D H(\omega):\left(\mathbb{C}^{m}, C_{\Omega, \omega}\right) \rightarrow\left(\mathbb{C}^{m}, C_{\Omega, \omega}\right)
$$

is an isometry then $\varphi_{\omega}^{(k)}(\boldsymbol{A}, \cdot)$ is contractive on $H^{\infty}(\Omega)$ if and only if $\varphi_{0}^{(k)}(\boldsymbol{A}, \cdot)$ is contractive on the algebra $H^{\infty}\left(\left(\mathbb{C}^{m}, C_{\Omega, \omega}\right)_{1}\right)$.

Proof: As we have pointed out, the operator norm on $\mathbb{C}^{k \times k}$ and the Carathéodory norm of $\left(\mathbb{C}^{k \times k}\right)_{1}$ at 0 coincide. If $F: \Omega \rightarrow\left(\mathbb{C}^{k \times k}\right)_{1}$ is holomorphic with $F(\omega)=0$ then $D F(\omega)$ : $\left(\mathbb{C}^{m}, C_{\Omega, \omega}^{*}\right) \rightarrow\left(\mathbb{C}^{k \times k}\right.$, op $)$ is a contraction, which is the norm decreasing property of the Carathéodory norm. This amounts to the inclusion

$$
\mathcal{D} \Omega^{(k)}(\omega) \subseteq\left(\left(\mathbb{C}^{m}, C_{\Omega, \omega}^{*}\right) \check{\otimes}\left(\mathbb{C}^{k \times k}, \mathrm{op}\right)\right)_{1}
$$


For any $\Lambda \in\left(\left(\mathbb{C}^{m}, C_{\Omega, \omega}^{*}\right) \check{\otimes}\left(\mathbb{C}^{k \times k}, \text { op }\right)\right)_{1}$, we see that the map

$$
G=\Lambda \circ H: \Omega \rightarrow\left(\mathbb{C}^{k \times k}, \mathrm{op}\right)_{1}
$$

is holomorphic. We can assume without loss of generality that $D H(\omega)=I$, therefore $D G(\omega)=\Lambda$. Thus we get the opposite inclusion in 1.12. In view of lemma 1.4 , the proof is complete.

\subsection{THE CASE OF A PRODUCT DOMAIN}

Let $\Omega_{1}, \Omega_{2} \subseteq \mathbb{C}$ be any two bounded domains, $\mathcal{P}=\Omega_{1} \times \Omega_{2}$ be the product domain in $\mathbb{C}^{2}$ and $\omega=\left(\omega_{1}, \omega_{2}\right) \in \mathcal{P}$ be an arbitrary but fixed point.

THEOREM 1.7 The homomorphism $\varphi_{\omega}(\boldsymbol{A}, \cdot)$ is completely contractive on $H^{\infty}(\mathcal{P})$ if and only if $\varphi_{0}(\boldsymbol{A}, \cdot)$ is completely contractive over the algebra $H^{\infty}\left(\left(\mathbb{C}^{2}, C_{\mathcal{P}, \omega}\right)_{1}\right)$. The norm $C_{\mathcal{P}, \omega}$ is a weighted $\ell^{\infty}$ norm.

Proof: $\quad$ For $j=1,2$, let $F_{\Omega_{j}, \omega_{j}}$ be the Ahlfors functions (cf. [3, page 109]) for the domains $\Omega_{j}$ at the points $\omega_{j} \in \Omega_{j}$. The unit ball with respect to the dual Carathéodory $\operatorname{norm}\left(\mathbb{C}, C_{\Omega_{j}, \omega_{j}}\right)_{1}$ is a disk of radius $r_{j}=D F_{\Omega_{j}, \omega_{j}}\left(\omega_{j}\right)$. Thus, $C_{\Omega_{j}, \omega}\left(v_{j}\right)=r_{j}\left|v_{j}\right|$. Let $G_{j}=r_{j}^{-1} F_{\Omega_{j}, \omega_{j}}$. Then $G_{j}: \Omega_{j} \rightarrow\left(\mathbb{C}, C_{\Omega_{j}, \omega_{j}}\right)_{1}$ and the derivative $D G_{j}\left(\omega_{j}\right)=1$. Since the Carathéodory norm of a product domain is the maximum of the Carathéodory norms on the factors (see [4]), it follows that

$$
C_{\mathcal{P}, \omega}\left(v_{1}, v_{2}\right)=\max \left\{r_{1}\left|v_{1}\right|, r_{2}\left|v_{2}\right|\right\}
$$

The map $G=\left(G_{1}, G_{2}\right)$ maps $\mathcal{P}$ into $\left(\mathbb{C}^{2}, C_{\mathcal{P}, \omega}\right)_{1}$. An application of Theorem 1.6 completes the proof.

Note that if $\Omega_{j}$ for each $j=1,2$ is bounded by a simple analytic closed curve then the Ahlfor's function is analytic in a neighbourhood of $\bar{\Omega}$ (see [3, Theorem 1.6, page 114]). So the Carathéodory norm remains unchanged if we use either rational functions or functions analytic in a neghbourhood of $\bar{\Omega}$ instead of the $H^{\infty}$ functions. Thus the theorem above can be used to study spectral and complete spectral sets.

EXAmple 1.8 Let $\Omega_{1}, \Omega_{2} \subseteq \mathbb{C}$ be any two bounded domains, $\mathcal{P}=\Omega_{1} \times \Omega_{2}$ be the product domain in $\mathbb{C}^{2}$ and $\omega=\left(\omega_{1}, \omega_{2}\right) \in \mathcal{P}$ be an arbitrary but fixed point. If $z \in\left(\mathbb{C}^{2}, C_{\mathcal{P}, \omega}\right)$, then

$$
C_{\mathcal{P}, \omega}(z)=\max \left(r_{1}\left|z_{1}\right|, r_{2}\left|z_{2}\right|\right)
$$

by Theorem 1.7. This theorem also implies that $\alpha(\mathcal{P}, \omega)=\alpha\left(\left(\mathbb{C}^{2}, C_{\mathcal{P}, \omega}\right)_{1}, 0\right)$. Since, $\left(\mathbb{C}^{2}, C_{\mathcal{P}, \omega}\right)_{1}$ is bi-holomorphic to the bi-disk, it follows via Ando's theorem that $\alpha(\mathcal{P}, \omega)=1$ for every $\omega \in \mathcal{P}$. 


\subsection{A SPECIAL CLASS OF HOMOMORPHISMS}

We now specialise to those domains $\mathcal{B}$, which can be realised as the unit ball with respect to some norm $\|\cdot\|_{\mathcal{B}}$ in $\mathbb{C}^{m}$. So far, we have considered $\boldsymbol{A}=\left(A_{1}, \ldots, A_{m}\right)$, where each $A_{i} \in \mathbb{C}^{n \times n}, i=1, \ldots, m$, is arbitrary. Suppose now that except for the first row every other row in $A_{i}, i=1, \ldots, m$, is zero. We will label this nonzero row in $A_{i}$ as $a_{i}$. Let $\boldsymbol{z}=\left(z_{1}, \cdots, z_{m}\right) \in \mathcal{B}$, and $b_{i}$ be vectors in $\mathbb{C}^{k}$ for $i=1, \ldots, m$.

TheOrem 1.9 For $\boldsymbol{A}$ as above, $\varphi_{0}^{(k)}(\boldsymbol{A}, \cdot)$ is contractive if and only if

$$
\sup \left\{\left|\sum\left\langle b_{i}, b_{j}\right\rangle\left\langle a_{i}, a_{j}\right\rangle\right|:\left\|z_{1} b_{1}+\cdots+z_{m} b_{m}\right\|_{2} \leq 1 \text { for all } \boldsymbol{z} \in \mathcal{B}\right\} \leq 1 .
$$

Proof: Let $\boldsymbol{B}=\left(B_{1}, \cdots, B_{m}\right), B_{i} \in \mathbb{C}^{k \times k}$ for $i=1, \ldots, m$ be such that

$$
\left\|z_{1} B_{1}+\cdots+z_{m} B_{m}\right\|_{\text {op }} \leq 1, \text { for all } z \in \mathcal{B}
$$

that is,

$$
\sup _{\boldsymbol{z} \in \mathcal{B}}\left\{\left|\left\langle\sum\left(\bar{z}_{i} z_{j}\right) B_{i}^{*} B_{j} \boldsymbol{x}, \boldsymbol{x}\right\rangle\right| \leq 1, \text { for } \boldsymbol{x} \text { in } \ell^{2}(k),\|\boldsymbol{x}\|_{2} \leq 1\right\},
$$

where $\ell^{2}(k)$ denotes $k$ dimensional complex Hilbert space. Or, equivalently the operator defined by

$$
\left(z_{1}, \ldots, z_{m}\right) \rightarrow z_{1} B_{1} \boldsymbol{x}+\cdots+z_{m} B_{m} \boldsymbol{x},
$$

is a contraction from $\mathbb{C}^{m}$ with the norm determined by $\mathcal{B}$ into $\ell^{2}(k)$ for each $\boldsymbol{x}$ in the unit ball of $\ell^{2}(k)$. The contractivity of $\varphi_{0}^{(k)}(\boldsymbol{A}, \cdot)$ is thus (see 1.11) equivalent to

$$
\left\|B_{1} \otimes a_{1}+\cdots+B_{m} \otimes a_{m}\right\|_{\mathrm{op}} \leq 1
$$

for $\boldsymbol{B}$ as above. Let $b_{i}=B_{i} \boldsymbol{x}, i=1,2, \ldots, m$. In this notation, 1.13 is equivalent to

$$
\begin{aligned}
1 & \geq \sup \left\{\left|\left\langle\sum B_{i}^{*} B_{j}\left\langle a_{j}, a_{i}\right\rangle \boldsymbol{x}, \boldsymbol{x}\right\rangle\right|: \boldsymbol{x} \in \ell^{2}(k),\|\boldsymbol{x}\|_{2} \leq 1\right\} \\
& =\sup \left\{\left|\sum\left\langle B_{i}^{*} B_{j}\left\langle a_{j}, a_{i}\right\rangle \boldsymbol{x}, \boldsymbol{x}\right\rangle\right|: \boldsymbol{x} \in \ell^{2}(k),\|\boldsymbol{x}\|_{2} \leq 1\right\} \\
& =\sup \left\{\left|\sum\left\langle b_{j}, b_{i}\right\rangle\left\langle a_{j}, a_{i}\right\rangle\right|:\left\|z_{1} b_{1}+\cdots+z_{m} b_{m}\right\|_{2} \leq 1 \text { for } \boldsymbol{z} \in \mathcal{B}\right\}
\end{aligned}
$$

This completes the proof.

The theorem specialised to $k=1$ says that the operator

$$
A=\left(\left(\left\langle a_{j}, a_{i}\right\rangle\right)\right):\left(\mathbb{C}^{m},\|\cdot\|_{\mathcal{B}}^{*}\right) \rightarrow\left(\mathbb{C}^{m},\|\cdot\|_{\mathcal{B}}\right)
$$

is a contraction. The operator

$$
B=\left(\left(\left\langle b_{j}, b_{i}\right\rangle\right)\right):\left(\mathbb{C}^{m},\|\cdot\|_{\mathcal{B}}\right) \rightarrow\left(\mathbb{C}^{m},\|\cdot\|_{\mathcal{B}}^{*}\right)
$$

is a contraction if and only if $\sup _{z \in \mathcal{B}}\left\|z_{1} b_{1}+\cdots+z_{m} b_{m}\right\|_{2} \leq 1$. In the following corollary $\|A\|,\|B\|$ are the operator norms of $A$ and $B$ respectively. 
COROLlary 1.10 The following two conditions are equivalent.

1. Every contractive homomorphism $\varphi_{0}(\boldsymbol{A}, \cdot)$ is completely contractive over $H^{\infty}(\mathcal{B})$ for $\boldsymbol{A}$ as in this section.

2. $\langle A, B\rangle \leq\|A\|\|B\|$, for all $A \geq 0 B \geq 0$, where $\langle\cdot, \cdot\rangle$ denotes the Hilbert-Schmidt inner product.

\section{Tensor Product Norms}

All Banach spaces considered in this note are finite dimensional complex spaces. For two such spaces $V, W$, we shall use the natural identification of $V \otimes W$ with the space of (linear) operators from $V^{*}$ into $W$. Thus viewed, the injective tensor product norm $\|\cdot\|$ on $V \otimes W$ is just the operator norm. The projective tensor product norm $\|\cdot\|$ is the norm on $V \otimes W$ dual to the injective norm on the dual space $W^{*} \otimes V^{*}$. By general nonsense, we have $\|\cdot\| \leq\|\cdot\|$. For computational purposes, we shall think of the elements of $V \otimes W$ as matrices with respect to standard bases. Finally, the complex space of dimension $n$ equipped with the $l^{p}$ norm $(1 \leq p \leq \infty)$ will be denoted by $l^{p}(n)$. T will denote the unit circle.

Note that there is a natural notion of positivity for elements of $V \otimes V$. Namely, an element $A$ of $V \otimes V$ is positive (written $A \geq 0$ ) if it is in the convex hull of the set of symmetric tensors $x \otimes x, x \in V$, equivalently, it is an element which factors as $A=B^{*} B$ for some $B \in V \otimes l^{2}$.

Because of its intimate connection with the 'spectral implies complete spectral' question for the class of operator tuples discussed above (see Corollary 1.10), we were led to ask : what are the spaces $V$ which satisfy the following

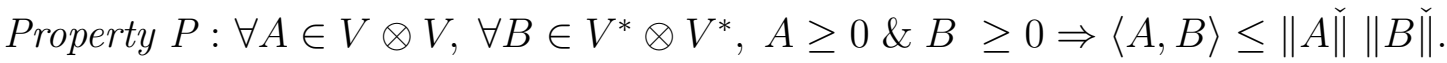

Here $\langle.,$.$\rangle is the Hilbert-Schmidt inner product.$

A stronger property which a Banach space may possess is :

Property $Q: \forall A \in V \otimes V, A \geq 0 \Rightarrow\|A\|=\| A \check{\|}$.

Fact 0 : Property $Q \Rightarrow$ Property $P$.

Proof: This is immediate from the definition of the projective norm as a dual norm.

Fact 1 : Property $P$ is conserved by duality but Property $Q$ is not so conserved. Thus, Property $P \nRightarrow$ Property $Q$. 
Proof: From the symmetry in the statement of Property $P$, it is clear that if $V$ has Property $P$ then so does $V^{*}$ (and, hence, conversely). We shall see that $l^{\infty}(3)$ has Property $Q$ but $l^{1}(3)$ does not have it. In consequence, $l^{1}(3)$ has Property $P$ though it does not have Property $Q$.

Recall that for $u \in V^{*} \otimes l^{2}$, the 2-summing norm $\pi_{2}(u)$ is defined as

$$
\left(\pi_{2}(u)\right)^{2}=\sup \left\{\sum_{i=1}^{n}\left\|u x_{i}\right\|^{2}\right\}
$$

where the supremum is over all $n \geq 1$ and all $x_{1}, \ldots, x_{n} \in V$ for which $\sum_{i=1}^{n}\left|x^{*}\left(x_{i}\right)\right|^{2} \leq 1$ for all $x^{*}$ in the unit ball of $V^{*}$.

From the case $n=1$ in the definition of $\pi_{2}(u)$, it is clear that we have $\pi_{2}(u) \geq \| u\lceil\forall u \in$ $V^{*} \otimes l^{2}$. In [2] $V$ is said to have the 2-summing property if $\pi_{2}(u)=\|u\| \forall u \in V^{*} \otimes l^{2}$.

Fact 2 : Property $P$ is equivalent to the 2 -summing property.

(Remark: This fact was brought to our attention by Pisier in a private conversation. But the proof presented below is more direct than the argument outlined by him.)

Proof: Given $x_{1}, \ldots, x_{n} \in V$, if we define $T \in l^{2}(n) \otimes V$ by $T e_{j}=x_{j}, j=1, \ldots, n$ (where $e_{j}$ are the standard basis vectors), then $T^{*}\left(x^{*}\right)=\sum_{j=1}^{n} x^{*}\left(x_{j}\right) e_{j}, x^{*} \in V^{*}$. Thus the condition (in the definition of $\left.\left.\pi_{2}\right)\right) \sum_{j=1}^{n}\left|x^{*}\left(x_{j}\right)\right|^{2} \leq 1$ for all $x^{*}$ in the unit ball of $V^{*}$ means that $\| T \check{\|} \leq 1$. But $\sum_{j=1}^{n}\left\|u x_{j} \check{I}^{2}=\sum_{j=1}^{n}\right\| u T e_{j} \check{\|}^{2}=\langle u T, u T\rangle=\left\langle u^{*} u, T T^{*}\right\rangle$. Hence, putting $S=T T^{*}$, we find that for $u \in V^{*} \otimes l^{2}$,

$$
\left(\pi_{2}(u)\right)^{2}=\sup \left\{\left\langle u^{*} u, S\right\rangle: S \geq 0, S \in V \otimes V,\|S\| \leq 1 .\right\}
$$

Thus Property $P$ says that $\pi_{2}(u) \leq\|u\|$ for all such $u$. We have already remarked that the reverse inequality is trivial.

Fact 3: $l^{1}(2)$ has Property $Q$.

Proof: $\quad$ Since $l^{\infty}(2) \check{\otimes} l^{\infty}(2)=l^{\infty}(4)$, we have, for $0 \leq A=\left(\begin{array}{cc}a & b \\ \bar{b} & c\end{array}\right) \in l^{1}(2) \otimes l^{1}(2)$, $\| A \hat{\|}=|a|+2|b|+|c|$. But

$\| A ॉ=\sup \{|a+b \omega|+|\bar{b}+c \omega|: \omega \in \mathbb{T}\}=\sup \{|a+b \omega|+|c+b \omega|: \omega \in \mathbb{T}\}=|a|+2|b|+|c|$

as is seen by taking $\omega=\bar{b} / b$.

(Remark : we have not used the full force of the hypothesis on $A$. See Lemma 2.1 below for an explanation.) 
Fact $4: l^{\infty}(2)$ has Property $Q$.

Proof: This follows from Facts 7 and 9 below.

Fact $5: l^{p}(2)$ does not have Property $P$ for $1<p<\infty$.

Proof: An easy norm computation shows that for $l^{p}(2), 1<p<\infty$, Property $P$ fails with both $A$ and $B$ taken as the $2 \times 2$ identity.

Lemma 2.1 Let $0 \leq A=\left(\begin{array}{ccc}* & \alpha & \beta \\ \bar{\alpha} & * & \gamma \\ \bar{\beta} & \bar{\gamma} & *\end{array}\right) \in l^{1}(3) \otimes l^{1}(3)$. Then, $\|A \hat{\|}=\| A \overline{\|}$ if and only if $\alpha \bar{\beta} \gamma$ is a non-negative real number.

(Here, and later, ${ }^{*}$ stands for an unspecified (real) number, not necessarily the same in all occurences.)

Proof: We may assume that $\alpha, \beta, \gamma$ are all non-zero (otherwise it reduces to Fact 3 ). Choose $\omega \in \mathbb{T}^{3}$ such that $\| A \check{\|}=\langle A \omega, \omega\rangle$. Now, $\|A \check{\|}=\| A \hat{\|}$ means that

$$
\operatorname{Re}\left(\alpha \omega_{1} \overline{\omega_{2}}+\beta \omega_{1} \overline{\omega_{3}}+\gamma \omega_{2} \overline{\omega_{3}}\right)=|\alpha|+|\beta|+|\gamma|
$$

This happens iff $a=\alpha \omega_{1} \overline{\omega_{2}}, b=\beta \omega_{1} \overline{\omega_{3}}, c=\gamma \omega_{2} \overline{\omega_{3}}$ are all non-negative reals. Now, this holds for some choice of $\omega \in \mathrm{T}^{3}$ iff $\alpha \bar{\beta} \gamma=a \bar{b} c \geq 0$.

Fact $6: l^{1}(3)$ does not have Property $Q$.

Proof: This is immediate from Lemma 2.1.

Fact $7: l^{\infty}(3)$ has Property $Q$.

Proof: Let $\mathcal{X}$ be the set of all $A \geq 0$ in $l^{\infty}(3) \otimes l^{\infty}(3)$ such that $\|A\| \leq 1$. Enough to show that for any extreme point $A$ of $\mathcal{X},\|A\|$ is $\leq 1$. Since $A$ is an extreme point of $\mathcal{X}, A \geq 0$ and all the diagonal entries of $A$ equal 1. Such an $A$ may be written as $A=U^{*} U$ where $U$ is upper triangular and all the columns of $U$ have $l^{2}$ length $=1$. Say,

$$
U=\left(\begin{array}{ccc}
1 & \alpha_{1} & \beta_{1} \\
0 & \alpha_{2} & \beta_{2} \\
0 & 0 & \beta_{3}
\end{array}\right)
$$

Hence, we have,

$$
A=\left(\begin{array}{ccc}
1 & \alpha_{1} & \beta_{1} \\
\overline{\alpha_{1}} & 1 & \overline{\alpha_{1}} \beta_{1}+\overline{\alpha_{2}} \beta_{2} \\
\overline{\beta_{1}} & \alpha_{1} \overline{\beta_{1}}+\alpha_{2} \overline{\beta_{2}} & 1
\end{array}\right)
$$


where $\left|\alpha_{1}\right|^{2}+\left|\alpha_{2}\right|^{2}=1,\left|\beta_{1}\right|^{2}+\left|\beta_{2}\right|^{2} \leq 1$.

Take a $B \in l^{1}(3) \otimes l^{1}(3)$ such that $\|B\| \leq 1$ and $B$ maximises $|\langle A, B\rangle|$ over all such matrices. Replacing $B$ by a suitable unimodular scalar multiple of $B$, we may assume $\langle A, B\rangle \geq 0$. But then $\left\langle A,\left(B+B^{*}\right) / 2\right\rangle=\langle A, B\rangle$ and $\left\|\left(B+B^{*}\right) / 2\right\| \leq\|B\| \leq 1$. So, replacing $B$ by $\left(B+B^{*}\right) / 2$ if necessary, we may assume that $B$ is self adjoint. Say,

$$
B=\left(\begin{array}{ccc}
* & \overline{\lambda_{1}} & \overline{\lambda_{2}} \\
\lambda_{1} & * & \overline{\lambda_{3}} \\
\lambda_{2} & \lambda_{3} & *
\end{array}\right)
$$

We have to show that $\langle A, B\rangle \leq \| B \check{\|}$. Since $\|B\| \geq \sup \langle B z, z\rangle$, where the supremum is over all $z=\left(\begin{array}{c}1 \\ \omega_{1} \\ \omega_{2}\end{array}\right) \in \mathrm{T}^{3}$, it is enough to show that $\langle A, B\rangle \leq \sup \langle B z, z\rangle$, that is,

$$
\operatorname{Re}\left(\lambda_{1} \alpha_{1}+\lambda_{2} \beta_{1}+\lambda_{3}\left(\overline{\alpha_{1}} \beta_{1}+\overline{\alpha_{2}} \beta_{2}\right)\right) \leq \sup _{\omega_{1}, \omega_{2} \in \mathrm{T}} \operatorname{Re}\left(\lambda_{1} \omega_{1}+\lambda_{2} \omega_{2}+\lambda_{3} \overline{\omega_{1}} \omega_{2}\right) .
$$

Since the left hand side is $\leq f\left(\alpha_{1}, \beta_{1}\right)$ where $f$ is as in Lemma 2.2, this follows from Lemma 2.2 below.

Lemma 2.2 Let $\lambda_{1}, \lambda_{2}, \lambda_{3}$ be complex numbers. Define the function $f$ on the closed bi-disc by :

$$
f(\alpha, \beta)=\operatorname{Re}\left(\lambda_{1} \alpha+\lambda_{2} \beta+\lambda_{3} \bar{\alpha} \beta\right)+\left|\lambda_{3}\right| \sqrt{\left(1-|\alpha|^{2}\right)\left(1-|\beta|^{2}\right)} .
$$

Then the maximum of $f$ is attained on the torus $\mathrm{T} \times \mathrm{T}$.

Proof: Rotating $\beta$, we see that at a maximum,

$$
f=\operatorname{Re}\left(\lambda_{1} \alpha\right)+|\beta|\left|\lambda_{2}+\lambda_{3} \bar{\alpha}\right|+\left|\lambda_{3}\right| \sqrt{\left(1-|\alpha|^{2}\right)\left(1-|\beta|^{2}\right)} .
$$

The last two terms here give the standard inner product between the two vectors $\left(|\beta|, \sqrt{1-|\beta|^{2}}\right)$ and $\left(\left|\lambda_{2}+\lambda_{3} \bar{\alpha}\right|,\left|\lambda_{3}\right| \sqrt{1-|\alpha|^{2}}\right)$. Therefore, Cauchy-Schwarz shows that at a maximum, these two vectors must be proportional (so that at a maximum, $|\alpha|=1 \Rightarrow|\beta|=$ 1.), and the maximum value of $f$ is

$$
\begin{aligned}
f & =\operatorname{Re}\left(\lambda_{1} \alpha\right)+\sqrt{\left|\lambda_{2}+\lambda_{3} \bar{\alpha}\right|^{2}+\left|\lambda_{3}\right|^{2}\left(1-|\alpha|^{2}\right)} \\
& =\operatorname{Re}\left(\lambda_{1} \alpha\right)+\sqrt{\left|\lambda_{2}\right|^{2}+\left|\lambda_{3}\right|^{2}+2 \operatorname{Re}\left(\lambda_{2} \overline{\lambda_{3}} \alpha\right)} .
\end{aligned}
$$

Therefore, it suffices to show that the maximum over $|\alpha| \leq 1$ of this last expression is attained at $|\alpha|=1$. Suppose, on the contrary, that the maximum is attained at a point $\alpha$ 
in the interior of the unit disc. Take $\omega,|\omega|=1$, such that $\lambda_{2} \overline{\lambda_{3}} \omega$ is purely imaginery. Then, replacing $\alpha$ by $\alpha \pm \epsilon \omega, \epsilon>0$, does not change the second term and the new value of $\alpha$ is in the unit disc provided $\epsilon$ is small. But the first term increases by $\pm \epsilon \operatorname{Re}\left(\lambda_{1} \omega\right)$, and, for the appropriate choice of sign, this increment is strictly positive if $\lambda_{1} \overline{\lambda_{2}} \lambda_{3}$ is not real. This contradiction proves that at a maximum for $f$ we must have $|\alpha|=1$ and hence also $|\beta|=1$, provided $\lambda_{1} \overline{\lambda_{2}} \lambda_{3}$ is not real. If, on the other hand, $\lambda_{1} \overline{\lambda_{2}} \lambda_{3}$ is real, then the above expression depends on $\alpha$ only through $\operatorname{Re}\left(\lambda_{1} \alpha\right)$. Since $|\alpha|$ can be increased to 1 without changing the value of $\operatorname{Re}\left(\lambda_{1} \alpha\right)$, we have the result in this case also.

Remark. The above Lemma is a minor variation of the 'calculus lemma' of [2]. In [2], Arias et. al. used the calculus lemma and a Hahn Banach argument to prove that $l^{\infty}(3)$ has Property $P$. We observed that their argument actually establishes the apparently stronger Property Q. (But, it turns out, in the case of $l^{\infty}(n)$, this appearance is illusory. See the following paragraph.) Arias et. al. [2] uses a sophisticated Hahn Banach argument to reduce this to the case of upper triangular matrices, and the calculus lemma does the rest. The point of the above proof is that a much more elementary reduction to the case of self adjoint matrices suffices, so that the calculus lemma carries the entire strength of the observation.

We show that if $l^{\infty}(4)$ had Property $P$, then it would have Property $Q$ as well. So assume it has Property $P$. Take $0 \leq A \in l^{\infty}(4) \otimes l^{\infty}(4)$, and $B \in l^{1}(4) \otimes l^{1}(4)$ such that all the diagonal entries of $A$ are $=1$ and $B$ is self adjoint. Arguing as in the proof of Fact 7, we see that, in order to deduce Property $Q$ for $l^{\infty}(4)$, it suffices to show that $\langle A, B\rangle \leq \sup \langle B z, z\rangle$ where the supremum is over all $z \in \mathbb{T}^{4}$. Take $r$ so large that $B+r I \geq 0$. Since $l^{\infty}(4)$ is supposed to have Property $P$, we get $\langle A, B\rangle+4 r=\langle A, B+r I\rangle \leq \sup \langle(B+r I) z, z\rangle=\sup \langle B z, z\rangle+4 r$, so that our claim follows.

A proof of the following fact is given in [2].

Fact $8: l^{\infty}(4)$ (and hence also $l^{1}(4)$ ) does not have Property $P$.

Recall that a subspace $W$ of a Banach space $V$ is called constrained if there is a projection of norm one from $V$ onto $W$.

Fact 9 : If a Banach space has Property P (or Q) then so does all its constrained subspaces.

Proof: Let $W$ be a constrained subspace of the space $V$. Let $i: W \rightarrow V$ be the inclusion map and $p: V \rightarrow W$ be a projection of norm 1. Let's define the maps $\phi: W \otimes W \rightarrow V \otimes V$ and $\psi: W^{*} \otimes W^{*} \rightarrow V^{*} \otimes V^{*}$ by $\phi(A)=i A i^{*}, \quad \psi(B)=p^{*} B p, \quad A \in W \otimes W, B \in W^{*} \otimes W^{*}$. It is easy to verify that these two maps preserve the injective norms. Also, together they preserve the inner product : $\langle\phi(A), \psi(B)\rangle=\langle A, B\rangle$. In consequence, they preserve the projective norms as well. Further, both maps preserve positivity. Therefore, these two maps pull back Property $P$ (or Q) from $V$ to $W$. 
Note that $l^{p}(m)$ is a constrained subspace of $l^{p}(n)$ for $m \leq n$. Therefore, the observations made above may be summarised in :

TheOrem 2.3 Let $n \geq 2$ be an integer and let $1 \leq p \leq \infty$. Then $l^{p}(n)$ has Property $\mathrm{Q}$ only for $(p, n)=(1,2),(\infty, 2),(\infty, 3)$. it has Property $\mathrm{P}$ but not $Q$ only for $(p, n)=(1,3)$.

Unfortunately, the only constrained subspaces of $l^{\infty}(n)$ (resply $\left.l^{1}(n)\right)$ are (isometric to) $l^{\infty}(m)$ (resply $\left.l^{1}(m)\right), m \leq n$. Therefore Fact 9 does not enable us to find more examples of 2-dimensional spaces with Property $P$ or Q. However, in [2] it is shown that every subspace of $\ell^{\infty}(3)$ has the 2 -summing property, that is, any such subspace has Property P. Arias et. al. [2] have also determined all real Banach spaces with Property $P$. In view of their result and the above theorem, it is perhaps not too wild a guess that $l^{\infty}(3)$ may be the only complex Banach space of dimension $\geq 3$ which has Property $Q$.

Parrott's example (cf. [6],[7]) shows that there is a contractive homomorphism $\varphi_{0}(\boldsymbol{A}, \cdot)$ on $H^{\infty}\left(\mathbb{D}^{3}\right)$, which is not completely contractive. This study was motivated partly to see if "Parrott like" examples can be found in the restricted class of homomorphisms considered in section 1.4. Theorem 2.1 answers this question in the negative. Further it follows that every contractive homomorphism $\varphi_{0}(\boldsymbol{A}, \cdot)$ on $H^{\infty}(\Omega)$, with $\boldsymbol{A}$ in the restricted class is completely contractive when $\Omega \subseteq \mathbb{C}^{3}$ is a product of three possibly different domains in $\mathbb{C}$ as well as domains $\Omega$ in $\mathbb{C}^{2}$ or $\mathbb{C}^{3}$ of the kind considered in Example 1.5 above.

\section{References}

[1] J. Agler, Operator theory and the Carathéodory metric, Inv. Math, 101(1990), 483-500.

[2] A. Arias, T. Figiel, W. B. Johnson, and G. Schechtman, Banach spaces which have the 2-summing property, preprint.

[3] S. D. Fisher, Function Theory on Planar domains, John Wiley \& Sons, 1983.

[4] M. Jarnicki and P. Pflug, The Carathédory pseudodistance has the product property, Math. Ann., 285(1989), 161-164.

[5] S. G. Krantz, Function Theory of Several Complex Variables, John Wiley \& Sons, 1982.

[6] G. Misra, Completely contractive Hilbert modules and Parrott's example, Acta. Math. Hungarica, 63(1994), 291-303. 
[7] V. I. Paulsen, Representations of Function algebras, Abstract Operator spaces, and Banach space Geometry, J. Functional Anal., 109(1992), 113-129.

[8] G. Pisier, Private Communications.

[9] W. Rudin, Function theory on the unit ball of $\mathbb{C}^{n}$, Springer Verlag, 1980.

Statistics and Mathematics Division

Indian Statistical Institute

Bangalore 560059

India 